\title{
The Message-Passing Interface and Parallel SAT-Solvers
}

\author{
Levis Zerpa \\ Yachay Tech \\ Hacienda San Jose s/n y Proyecto Yachay \\ San Miguel de Urcuqui, Imbabura, Ecuador 100650 \\ lzerpa@yachaytech.edu.ec
}

\begin{abstract}
In this paper we examine the communication aspects of parallel SAT-solving. In particular, the paper is focused on the system PMSat. Besides the algorithms and heuristics implemented in that system, PMSat implements the Message-Passing Interface, the standard for a huge range of parallel architectures. A detailed analysis of some of the source code of the system shows some interesting and non-trivial aspects of the MPI functions in the treatment of the SAT problem. Moreover, the advantage of the use of the RMA operations from MPI-2 is the possibility of taking advantage of the direct semantic matching of these functions with the RDMA operations of InfiniBand and other interconnect networks.
\end{abstract}

\section{CCS CONCEPTS}

- Computing methodologies $\rightarrow$ Boolean algebra algorithms; Parallel programming languages; • Applied computing $\rightarrow$ Mathematics and statistics

\section{KEYWORDS}

Boolean Satisfiability, Message-Passing Interface, Remote Memory Operations

\section{ACM Reference format:}

Levis Zerpa. 2017. The Message-Passing Interface and Parallel SAT-Solvers. In Proceedings of ICFNDS '17, Cambridge, United Kingdom, fuly 19-20, 2017, 7 pages.

https://doi.org/10.1145/3102304.3102336

\section{INTRODUCTION}

In this paper we examine the communication aspects of parallel SATsolving. Being a demonstrably hard problem, [1], in the last forty years there has been a substantial improvement in the serial and parallel algorithms and heuristics used; it has been also a growing development of parallel SAT solvers [9], [10]. Now, an effective use of parallel computing architectures is based on the efficiency of two aspects: the local computations and the interconnection network communication. However, it is often the case that communication aspects are not explicitly considered and adequately appreciated.

Permission to make digital or hard copies of all or part of this work for personal or classroom use is granted without fee provided that copies are not made or distributed for profit or commercial advantage and that copies bear this notice and the full citation on the first page. Copyrights for components of this work owned by others than ACM must be honored. Abstracting with credit is permitted. To copy otherwise, or republish to post on servers or to redistribute to lists, requires prior specific permission and/or a fee. Request permissions from permissions@acm.org.

ICFNDS '17, July 19-20, 2017, Cambridge, United Kingdom

(C) 2017 Association for Computing Machinery.

ACM ISBN 978-1-4503-4844-7/17/07 . \$15.00

https://doi.org/10.1145/3102304.3102336
This paper is focused in the study of a particular parallel SAT solver: PMSat, [4]. This solver implements the message passing model of parallel computation. However, in the paper on PMSat, [4], there is no analysis or even explicit discussion of the communication aspects. In contrast, this paper is focused on the communication patters contained in PMSat. In fact, we examine the implementation of message passing through the use of MPI functions in the source code of PMSat. But we also go beyond PMSat and restate its communication patters using remote memory access (RMA) operations, [13] (chap. 3). In contrast with message passing, RMA operations allows a more efficient use of parallel hardware because of two main reasons: first, unlike message passing (consisting of two-sided communication routines), the program running in the remote process does not need to call any routines to match the information sent; actually, the sending process has direct access to the memory in the remote process (one-sided communication routines). This feature simplify the code in a truly remarkable way and allows us to see the logical structure of the communication patterns in a clearer form. Second, and very importantly, interconnection networks provide a direct implementation of such RMA operations and this improves the systems in a substantial way. For example, InfiniBand, [5], the interconnection network used in the $65 \%$ of supercomputers included in the TOP500 lists (according to Mellanox, [7] ${ }^{1}$ has RDMA operations, so MPI-2 RMA operations are directly implementable in the InfiniBand network. Several designs have been proposed to take optimal advantage of the MPI-2 and MPI-3 RMA operations in the context of InfiniBand technology. For example, Jiang et al, [6] propose a high performance MPI-2 one-sided communication design over the InfiniBand Architecture. In their design, MPI-2 RMA operations such as MPI_Put, MPI_Get and MPI_Accumulate are directly mapped to InfiniBand Remote Direct Memory Access (RDMA) operations. These reasons motivates the improvement of the communication patterns implemented by PMSat by means of the RMA operations of the MPI- 2 and the MPI-3 standards.

The organization of the paper is the following: an introductory section discussed some basic notions of parallel computing and related notions. Then the main ideas and algorithms from PMSat are discussed in detail. In particular, the functions of send and receive in MPI are introduced in the context of the overall architecture of the system. Next, the use of the MPI functions in the source code of one of the most important components of the system, namely, the master's management function, is discussed in detail. The analysis shows some of the non-trivial aspects of communication in PMSat and other similar systems.

\footnotetext{
${ }^{1}$ In fact, the fastest supercomputer, the Sunway, uses InfiniBand (according to Dongarra's Report, [3]).
} 


\section{THE BOOLEAN SATISFIABILITY PROBLEM)}

The Boolean Satisfiability Problem (SAT) is one of the most researched problems in Computer Science, [9], [10]. The problem is concerned with the task of finding satisfying assignments to boolean formulas of propositional logic. It is the first NP-complete problem ever found, [1], and its applications range from industry to science. There has been a large number of improvements in many aspects; for example, more powerful heuristics and faster algorithms have been discovered and implemented in a large number of SAT-solvers. Another substantial improvement has been the study of the problem on parallel architectures.

The parallelization of an algorithm involves the consideration of calculation techniques and communication interfaces. Sometimes the emphasis is placed on the calculation and less attention is focused on the communication aspects. As a matter of fact, one of the most interesting parallel SAT-solvers, PMSat, [4], is based on very efficient heuristic techniques and the Message-Passing Interface (MPI) as communication platform but this last component is not studied in detail in the paper of Gil et al. [4]; a similar consideration can be made with respect to other SAT-solvers. However, the aspects of communication in the computational treatment of the SAT problem bring interesting and sometimes difficult challenges and the MPI standard is particular appropriate framework to discuss them. In this article we focus the attention in the communication aspects of PMSat, [4], which implements MPI.

\section{PARALLEL COMPUTING}

From the point of view of hardware technology, the constant availability of faster and cheaper microprocessors (Moore's law) has been crucial for the creation of parallel computers. From the point of view of software technology, the development of Unix, Linux and other powerful operating systems that support parallel hardware have been crucial for the grow of parallel computing. At the same time, the improvement in the performance and capacity of both local area and wide area networks (LANs and WANs) makes possible to build applications that spans the world, [12] and [2].

The diverse parallel architectures proposed go from virtual clusters (like Amazon's EC2), Beowulf clusters, [11], to extremely fast supercomputers like the Sunway TaihuLight System, [2] (chaps. 1 to 3), [3]. A Beowulf cluster is a multi-computer architecture consisting of a server node and one or more clients nodes connected together through Ethernet or other fast network. In contrast, supercomputers have a much complex architecture, [2].

Several models of parallel computation have been developed but the message passing model have prevailed in a huge number of applications. It is important to keep in mind that MPI is only a definition for an interface; diverse vendors have develop their own implementation like MPICH. As the members of the MPI Forum say, "the attractiveness of the message-passing paradigm at least partially stems from its wide portability." [12].

A remarkable fact is that MPI has become the de-facto method of writing message-passing applications in both the academy and the industry, and MPI runs in all the spectrum of the parallel architectures, from the smaller clusters to the most powerful supercomputers. This fact is the more remarkable considering that before the 1990's no standard was established to develop parallel applications based on the message passing model. In 1994 the situation change with the interface and standard defined as MPI-1.0. Soon, in 1997 MPI-2.0 was released and the standard have evolved with many extensions and clarifications until MPI-3.1, the last version, introduced in June of 2015, [8]. Today the MPI standard supports point-to-point message-passing, collective communications, and many other features. Language bindings for $\mathrm{C}, \mathrm{C}++$ and Fortran are defined.

\section{MAIN IDEAS AND ALGORITHMS FROM PMSAT}

As we mention in the introduction, the paper in which PMSat is described, [4], does not contain any specific mention or analysis of the MPI routines used. For that reason, it is particularly appropriate to analyse and explain the use of the main MPI routines in the source code of PMSat.

\subsection{Decomposition of the SAT Problem}

The design of a parallel algorithm usually starts with a choice between two main decomposition procedures: domain or data decomposition and functional (or control) decomposition. Domain decomposition is a method of partitioning a large problem domain into smaller problems; basically it is a version of the "divide and conquer" technique. In contrast, functional decomposition consists in splitting the algorithm (the tasks or instructions) instead of the data in independent procedures that can be executed in parallel, [2, p. 47] and [4, p. 76]). The architecture of the main algorithms used in this area, [4], discourages domain functional decomposition because the correct executions of those algorithms depends on the coherence in the data structures which leads to a sequential update of the various functions. In contrast, domain decomposition can be implemented in a much more natural, easy, and efficient way.

Basically a SAT solver searches through a combinatorial space for an assignment that satisfies all clauses. Once such an assignment is identified, the search is halted and a result of SAT is returned. If no such assignment exists, the solver implicitly tries all combinations returning a final result of UNSAT (but conflict detection and clause learning guarantees that this search is never exhaustive). An interesting fact is that multiple combinations of assignments that compose the search can be represented as a binary tree (an asignment tree) where all branches and subtrees are independent of each other, allowing their exploration in parallel. Such exploration in parallel is implemented using some kind of parallel programming model. One of the simpler and most popular parallel programming models is the one implemented in PMSat: the master-worker or Task Farm approach explained in the next subsection.

\subsection{Architecture and Work Flow of the PMSat Parallel Algorithm}

The main idea of the the master-worker model is that one process, called the master, is responsible for coordinating the work of all the other processes, called the workers. This programming prototype is particularly appropriate for situations in which the workers do not have to communicate with one another but only with the manager. This simple model does not lack serious drawbacks ([2, p. 46]) but 
still constitutes an interesting step in the parallel approach to the SAT problem.

The very basic calculation and communication activities of the master and the workers in PMSat can be summarized in the following two steps:

(1) Each worker receives from the master a set of assumptions that need to be tested for satisfiability.

(2) The workers explore the search subspace that arise from those assumptions and return back a result to the master.

In PMSat the work flow can be summarized in the following way ([4, pp. 78-79]):

(1) upon starting, the program detects whether it is either a master or a worker (rank or ID 0 is assigned to the master and $1,2, \ldots$ to the workers);

(2) at the beginning, the program requires the user to choose between certain modes of operation; once the user's choice is entered, the master partitions the search space according to the mode selected. This step corresponds to the generation of the set of assumptions which are sent to the workers; after this, the master waits for results from the workers;

(3) the workers search for satisfiability using the assumptions generated in the previous step; this search is carry out in the subspace implicitly defined by these assumptions;

(4) if a worker returns UNSAT, then it may send a vector with a set of learnt clauses or a set of conflicts or both with at least some of the assumed literals responsible for that result. After this the worker waits for instructions from the master;

(5) after receiving the vector of conflicts the master use it to remove all untested assumptions (that will result in UNSAT);

(6) once the master receives an UNSAT result and updates the remaining set of assumptions, he sends another assumption with or without extra learnt clauses to the idle worker;

(7) when a worker returns SAT, it may send back the solution found;

(8) the execution ends when the master receives SAT or all the assumptions are reported as UNSAT.

Undoubtedly, the generation of assumptions is one of the most important features of PMSat. First at all, assumptions in PMSat are simply sets of literals assumed as true. A main strategy used in PMSat consists in the use of assumptions over a predefined, small set of variables and then searching for satisfiability on subtrees of the assignments trees using a standard solver. In the case of miniSAT, there is no need to change the underlying solver because it can received a vector with assumptions and determine whether the problem is SAT or UNSAT under those assumptions. (i.e., it can be directed to a specific subspace of the original space). Once the search is finished, control returns to the main solver, the assumptions are undone and the solver is ready to be used.

Most of the communication between master and workers are implemented by calls to the MPI functions send and receive. The next section are focused on those functions in a general way and the subsequent section analyses with great detail the communication exchanges between the master and the workers (the calculational tasks are also indicated but with much less detail). The purpose of such analysis is to find the communication patterns in the PMSat algorithm and their implementation by means of the MPI functions.

\section{MPI SEND AND RECEIVE}

The source code of PMSat has many calls to several MPI functions; a good starting point to analyze the use of the MPI interface in PMSat is to to consider first a minimal message-passing interface from the message-passing model ([12, sec. 2.3]) and then extends it to the implementation details involved in PMSat's source code.

The message-passing model of parallel computation is based on a fundamental fact: the processes executing in parallel have separate address spaces. In this context, communication occurs when a portion of one process's address space is copied into another process's address space. This operation is cooperative and occurs in one situation, namely, when the first process executes a send operation and the second process executes a receive operation. From this starting point one can first consider, in a general way, the information that should be considered by both the sender and the receiver and then the arguments of the functions send() and receive() in a more specific way from the point of view of the minimal message interface.

On the sender's side, the relevant information is clearly the data to be communicated and the destination process to which the data is to be sent. In contrast, on the receiver's side, the relevant information is the data to be received, the indication of the place in local memory in which such data will be stored, and the identity of the sender. Now, having established the relevant information on each side, which are the arguments of the send and receive functions? A minimal way to describe any kind of data is to specify a starting address in the parameter address and a length in bytes in length. Moreover, the destination can be described in different ways. An integer is enough to describe our third argument: destination. Until this point of the discussion, the send function has the following form: send(address, length, destination).

On the receiver's side, the minimum arguments are the address and length of an area in local memory where the received variable is to be stored. The identity of the sender or source is also required, so the receiver can identify which process sent the message. Consequently, the receive function has the form receive(address, length, source). However, an important feature is missing from this minimal interface, [12]; a process must be able to control which messages it receives and to form a queue of them. But this feature requires some form of matching between messages. By assigning an integer called tag to each message, a receive operation specifying a tag will complete successfully only when a message sent with a matching tag arrives. Therefore, tag is a basic parameter of both functions and through it the receiver process can identify the messages received. Finally, parameter actlen of the receive function specifies the length of the message received; the purpose of this argument is to specify a maximum message size (but shorter messages are allowed to arrive). In summary, the minimal interface becomes

send(address, length, destination, tag)

and

receive(address, length, source, tag, actlen). 
Two additional facts can also be considered. The message to be sent could be not contiguous (the data could be stored in noncontiguous buffers) and often machines represent information in different ways (for example, different machines use diverse byte ordering). Consequently. an MPI message buffer is defined by the triple (address, count, datatype) with count occurrences of the data type datatype starting at the address specified in address. In fact, a great advantage of the MPI library is that both elementary and user-defined datatypes can be used with the argument datatype.

A further consideration is the fact that processes belong to groups. So, in a group with $n$ processes, they are identified with ranks (integers from 0 to $n-1$ ). Finally, MPI contains communication contexts allocated at run time by the system (in response to requests from the user of the library). Both concepts of groups of processes and contexts are combined in the concept of communicator; this constitutes the last and sixth argument of the basic (blocking) operation send in MPI:

MPI_Send(address, count, datatype, destination, tag, comm)

where the first three arguments describe count occurrences of items of the type datatype starting at address, destination is the rank of the destination in the group associated with the communicator comm, and tag is an integer used for message matching. It is important to remember that a communicator identifies a group of processes and a communication context.

Furthermore, the operation receive becomes

MPI_Recv(address, maxcount, datatype, source, tag, comm, status)

where the triple (address, maxcount, datatype) describes the receive buffer in a way similar to MPI_Send. tag and comm are as in MPI_Send; source is the rank of the sender of the message in the group associated with the communicator comm and, finally, status contains information about the actual message size, source, and tag. Notice that parameters tag and source allow the use of wildcards like MPI_ANY_TAG and MPI_ANY_SOURCE. The default communicator (the only used in PMSat) is MPI_COMM_WORLD.

In a way partially similar to the concept of adequate set of connectives in propositional logic, the set $\{$ MPI_Init, MPI_Comm_size, MPI_Comm_rank, MPI_Send, MPI_Recv, MPI_Finalize $\}$ is adequate to write complete message-passing programs. As the names indicate, MPI_Init initializes MPI, MPI_Finalize terminates MPI, MPI_Comm_size finds out how many process exist and MPI_Comm_rank finds out which process I am.

MPI supports Fortran and $\mathrm{C}$ while MPI-2 supports Fortran90 and $\mathrm{C}++$. PMSat was written in $\mathrm{C}++$ and the $\mathrm{C}$ bindings for the send and receive routines are the following: ([12, p. 39]):

int MPI Send(const void *buf, int count, MPI Datatype datatype, int dest, int tag, MPI Comm comm), int MPI Recv(void *buf, int count, MPI Datatype datatype, int source, int tag, MPI Comm comm, MPI Status *status).

\section{ANALYSIS OF THE MASTER'S MANAGEMENT FUNCTION IN PMSAT FROM THE MPI PERSPECTIVE}

In this section the master's management function of PMSat is analyzed from the point of view of MPI. As we mention in the introduction, the original PMSat paper explains in a very clear way the algorithmic and heuristic aspects of the SAT-solver beyond the DP algorithm. However, the PMSat paper does not include any remark concerning the MPI implementation of the system, in spite of the fact that the PMSat source code is entirely based on the MPI library and the authors emphasize the role of MPI in their system. Moreover, the routines one finds in the source code are not always of an elementary nature. For example, the nonblocking MPI_Isend and MPI_Irecv functions as well as functions like MPI_Iprobe are found in the code. Therefore, following the miniSAT approach, it is convenient to spell out the MPI aspects of PMSat. But PMSat is a large program, so it is advisable to focus the attention in a specific component of the whole program. We choose the master's management function, test4SAT() (described in section 3.2 of [4] and lines 395 and ff. of the main file: Main. C) because this function contains several interesting aspects from the perspective of the message-passing interface.

\section{ANALYSIS OF TEST4SAT()}

In what follows we describe the structure of the the boolean function test4SAT() (the master's management function) in natural language with indications of the MPI functions called in parenthesis.

The function test4SAT() works in the following way: it first generates all the assumptions (and store them in the database $\mathrm{db}$ of assumptions). Then the function sends one assumption for each worker (this blocking sending is done with MPI_Send; the first argument, hyps, stores the address of the corresponding assumption). Then begins the main loop of the function; this loop terminates when all the assumptions are tested. The body of the loop consists of a first unconditional action and four conditional actions:

(1) Receives a result from a worker (with calls to MPI_Probe and MPI_Recv); if the result is SAT, then the system returns the message "CPU \% $d$ found the solution" where $d$ refers to the solver.

(2) If the learnt clauses are shared (that is, if the corresponding option is active and there is data to receive), then the master receives the learnt clauses and add them into the database $\mathrm{db}$ (with calls to the nonblocking MPI_Iprobe, MPI_Get_count and MPI_Recv).

(3) If there are assumptions that contain conflicting literals, remove them.

(4) If there are more assumptions to try, the share mode is selected, and if there are learnt clauses to send, then retrieve from database $\mathrm{db}$ a set of learnt clauses and send it (with 
MPI_Send; at this point the system shows the message "Master is sending learnt clauses to CPU \%d..." where "\%d" refers to the corresponding worker).

Finally, the function returns UNSAT and terminates. The relevant parts of the source code of this function are the following:

bool test4SAT(...)\{ // sending requests

... /* generate all asumptions: */

if (opts. shareLearnts)

$\mathrm{db}=$ new LearntsDB (cpus, ...);

...

do $\{\ldots$... * send one assumption from each worker: */

hyps = gen->nextAssumption();

MPI_Send(hyps, opts.nVars, MPI_INT, workerNumber,

JOB_TAG, MPI_COMM_WORLD);

workerNumber $=$ (workerNumber +1$) \%$ cpus;

... \}while(workerNumber $!=0$ \&\& gen->moreAssumps2Try());

/* MAIN LOOP OF THE FUNCTION */

do\{...//waits for an answer

MPI_Probe(MPI_ANY_SOURCE, RESULT_TAG, MPI_COMM_WORLD, \&status);

workerNumber = status.MPI_SOURCE;

I*receives all the messages from the worker possibly containing conflictuous literals: */

do\{

MPI_Recv(response, 1, typeResult, workerNumber, RESULT_TAG, MPI_COMM_WORLD, \&status);

$/ *$ if present, adds the conflicting literals to

conflictlist: */

for $(i=0 ; i<\operatorname{response}[0]$. conflictsize; $i++)\{$

conflictlist.push (response[0]. conflict[i]);

\}

\}while(response[0].moreMsgs);

...

break;

\}

received++;

/* removes the assumptions that contain the conflict

literals: */

if(opts. conflicts \&\& conflictList.size() $>0)\{$

int rem = gen->removeConflicts (conflictList);

... \} // if there are more work to send: */

if ( gen->moreAssumps2Try() ) \{

I* sends learnt clauses, to the same worker,

if in the share mode and there is data to send: */

if (opts. shareLearnts) \{

learnts $=\mathrm{db}$->getLearnts (workerNumber, learntsSize);

if (learnts $!=$ NULL $)\{$

...

MPI_Send(learnts, learntsSize, MPI_INT, workerNumber, LEARNT_TAG, MPI_COMM_WORLD) ; . . . \} \}

As we can see, at the beginning of the main loop of the function test4SAT() there is a call to MPI_Probe. This function performs a blocking test for a message and it can be used to query the message size before actually receiving it ([12, chap. 5]). The situation is the following: In many cases we are interested in send messages in which their length is known in advance. But in many other cases it is not possible; so we need to find out about a message before we receive it. The function MPI_Probe allows us to do this, making it possible to implement a dynamic form of receiving. MPI_Probe takes a source, tag and communicator and returns an MPI_Status in its last argument. PMSat waits for an answer from any worker (after the previous sending of the assumptions) executing

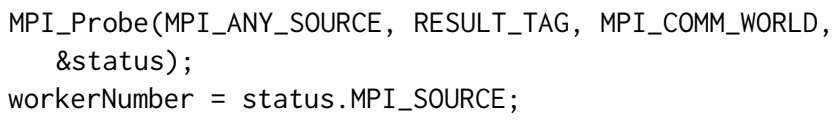

here the system gets the source from the MPI_Status returned by MPI_Probe; such source is stored in workerNumber and passed to the next call to MPI_Recv; the effect is that the system receives all the messages from the worker (previously identified).

Notice that the for loop adds the conflicting literals and then prepares the system to receive learnt clauses. Furthermore, as we mention before, the three functions MPI_Iprobe, MPI_Get_count and MPI_Recv (in this order) are used in certain parts of the code. MPI_Iprobe begins performing a nonblocking test and informs the master about the value of a flag before receiving the actual message. In the MPI_Status returned by MPI_Iprobe, just as if the actual message had been received, are two pieces of information: the message tag and the source. Then the call to MPI_Get_count gets the length of the message and MPI_Recv uses this information in its execution. In summary, through MPI_Iprobe and MPI_Get_count the function MPI_Recv will execute a dynamic receive.

\section{RMA BASIC OPERATIONS FROM MPI-2}

As it was mentioned in the introduction, there are two main approaches for communicating data between processes: point-to-point message passing and remote memory access operations. While the MPI-1 standard provides a powerful interface for the messagepassing approach, the MPI- 2 and MPI- 3 standards added RMA operations. As it was mentioned, interconnection networks like InfiniBand provide a direct implementation of such RMA operations and this improves the systems in a substantial way. The wide availability of these networking technologies (65\% of supercomputers included in the TOP500 lists, according to data provided by Mellanox, uses InfiniBand) motivates the study of the communication aspects of SAT solvers like PMSat.

Now, instead of moving data from one process to another with pairs of send and receive routines, RMA operations move data with as single routine that simply specifies where the data is coming from and where is going to. An RMA operation specifies both the send buffer and the receive buffer. In contrast with message passing, RMA operations do not require any matching between the sender and the receiver.

The RMA model involves three main steps:

(1) Define a memory window which is the memory of the process that can be used for the RMA operations; the MPI function MPI_Win is in charge of this step.

(2) Call MPI-2 functions MPI_Put, MPI_Get, or MPI_Accumulate to put data to, get data from, or to update data at the remote process, respectively (the MPI-3 variations of these functions like MPI_Rput, etc. can be used too). 
(3) Complete the data transfer. There are several ways to perform this operation but a simple and effective way is this: any pair of successive calls to MPI_Win_fence define a communication phase of the program. In fact, the function MPI_Win_fence serves as a barrier separating RMA accesses (raised from calls to MPI_Put, MPI_Accumulate, etc.) from non-RMA accesses.

As Gropp et al., [13, p. 71], point out, "MPI_Win_fence completes any RMA operation that started since the last call to MPI_Win_fence and ensures that any local stores to the memory window will be visible to RMA operations.... Developing an example from them, [13](pp. 59-61), we can compare two sequences of pseudocode (mixed with relevant fragments of actual code) in which the matching of the nonblocking sending and receiving of the data in the message-passing model corresponds to a single call to the RMA function MPI_Put in the remote memory access model. The two blocks of code are the following:

The MP block of code:

Define communicator

(object MPI_COMM_WORLD)

/* Master process $\theta$ sends and process 1 receives: */

if (rank == 0 ) \{ MPI_Isend(outbuf, ..., \&request);

\}

else if (rank == 1) \{

MPI_Irecv(inbuf, ..., \&request);

\}

Other operations (communication or computation)...

/* Complete the operation: */

MPI_Wait(\&request, MPI_STATUS_IGNORE;

/* Free the communicator: */

MPI_Comm_free(\&comm);

The RMA block of code:

Define memory window

(object MPI_Win)

/* Active a barrier: */

MPI_Win_fence $(0$, win);

I* Process $\theta$ sends and process 1 receives: * /

if ( $r$ ank $==0$ )

MPI_Put (outbuf, ..., win);

Other operations (communication or computation)...

/* Deactive the barrier: */

MPI_Win_fence $(\theta$, win);

/* Free the window: */

MPI_Win_free(\&win);
As one can see, the memory window in the RMA model serves approximately the role of the communicator in message passing. The function MPI_Put, like MPI_Isend, initiates a data transfer and MPI_Win_fence completes such data transfer in a similar way that MPI_Wait completes the transfer initiated by MPI_Isend. The asymmetry between the MP and RMA models (one sided versus two-sided communication routines) leads to the two following semantical matching, [6]:

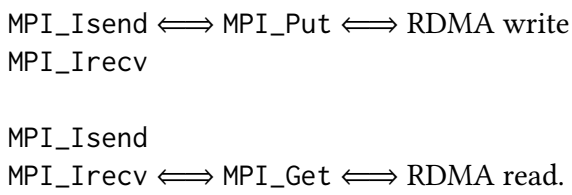

These ideas illustrate the way one can reformulate the master's function from PMSat using RMA operations. Once again, the great practical advantage of this reformulation from the point of view of networking technology is the direct mapping of such MPI functions in terms of the InfiniBand RDMA operations.

\section{CONCLUSIONS}

The analysis of the master's management function shows the type of considerations involved in the use of MPI as main communication interface for parallel SAT-solvers. Furthermore, the detailed analysis of part of PMSat's source code shows subtle and non-trivial aspects of MPI functions in the context of parallel SAT-solving. The advantage of the use of RMA operations is the possibility of taking advantage of the direct semantic matching of these functions with the RDMA operations of InfiniBand.

\section{ACKNOWLEDGMENTS}

The author would like to thank Dr. Yuefang Deng for his immense help during the workshop on high-performance computing held at Yachay E.P. (UrcuquÃy, Imbabura, Ecuador, march of 2017) as well as IBM Ecuador and Yachay E.P. and Yachay Tech for the opportunity to use the Quinde Supercomputer before its official opening (done on May 19, 2017).

\section{REFERENCES}

[1] Stephen A Cook. 1971. The complexity of theorem-proving procedures. In Proceedings of the third annual ACM symposium on Theory of computing. ACM, 151-158.

[2] Yuefan Deng. 2013. Applied parallel computing. World Scientific, Hackensack, New Jersey.

[3] Jack Dongarra. 2016. Report on the Sunway TaihuLight System. PDF). www. netlib. org. Retrieved fune 20 (2016).

[4] Luis Gil, Paulo Flores, and Luis Miguel Silveira. 2008. PMSat: a Parallel Version of MiniSAT. Journal on Satisfiability, Boolean Modeling and Computation 6 (2008), 71-98.

[5] InfiniBand Trade Association. 2016. InfiniBand Architecture Specification Volume 2. (Nov. 2016). Retrieved June 7, 2017 from https://cw.infinibandta.org/document/ $\mathrm{dl} / 7141$

[6] Weihang Jiang, Jiuxing Liu, Hyun-Wook Jin, Dhabaleswar K Panda, William Gropp, and Rajeev Thakur. 2004. High performance MPI-2 one-sided communication over InfiniBand. In Cluster Computing and the Grid, 2004. CCGrid 2004. IEEE International Symposium on. IEEE, 531-538.

[7] Mellanox Technologies. 2017. TOP500. (June 2017). Retrieved June 7, 2017 from http://www.mellanox.com/solutions/hpc/top500.php

[8] Message Passing Interface Forum. 2015. MPI: A Message-Passing Interface Standard - Version 3.1. (June 2015). Retrieved April 7, 2017 from http://mpi-forum. org/docs/mpi-3.1/mpi31-report.pdf 
[9] Daniel Singer. 2006. Parallel Resolution of the Satisfiability Problem: A Survey. Parallel Combinatorial Optimization (2006), 123-148.

[10] Julian Stecklina Peter Steinke Steffen Hölldobler, Norbert Manthey et al. 2011. A Short Overview on Modern Parallel SAT-Solvers. In Advanced Computer Science and Information System (ICACSIS), 2011 International Conference. IEEE, 201-206.

[11] Thomas Sterling. 2002. Beowulf Cluster Computing with Linux. The MIT Press, Cambridge, Massachusetts.

[12] Ewing Lusk William Gropp and Anthony Skjellum. 2014. Using MPI: Portable Parallel Programming with the Message-Passing Interface. The MIT Press, Cambridge, Massachusetts

[13] Rajeev Thakur William Gropp, Torsten Hoefler and Ewing Lusk. 2014. Using Advanced MPI: Modern Features of the Message-Passing Interface. The MIT Press, Cambridge, Massachusetts. 